\title{
Common Fixed Point Theorem for Semi-Compatible and Sub Compatible Maps in Fuzzy Metric Space
}

\author{
Sonal Dani and Sanjay Sharma
}

\begin{abstract}
The object of this paper is to introduce the concept of a pair of semi-compatible self-maps and sub compatibility in fuzzy metric spaces In this paper, we also introduce the notion of M-maps with respect to a single map and a pair of maps in fuzzy metric spaces and obtain common fixed point theorems for two pairs of sub compatible and semi compatible maps satisfying implicit relations.
\end{abstract}

Index Terms-Common fixed point theorem, fuzzy metric space, semi-compatibility, sub-compatibility, set valued M-maps.

\section{INTRODUCTION}

Zadeh introduced the concept of fuzzy sets in 1965 and [1] introduced the concept of fuzzy metric space in 1975. [2] modified the concept of fuzzy metric space introduced by [1]. Later several authors [3]-[8] etc. obtained fixed and common fixed point theorems satisfying various contractive conditions in fuzzy metric spaces.

[9] introduced the notion of semi-compatible maps in a $d$-topological space. They define a pair of self-maps $(S, T)$ to be semi-compatible if conditions (i) $S y=T y$ implies that $S T y=T S y$; (ii) for sequence $\left\{x_{n}\right\}$ in $\mathrm{X}$ and $x \in X$, whenever $\left\{S x_{n}\right\} \rightarrow x,\left\{T x_{n}\right\} \rightarrow x$, then $\left\{S T x_{n}\right\} \rightarrow T x$, as $n \rightarrow \infty$, hold. However, in Fuzzy metric space (ii) implies (i), taking $x_{n}=y$ for all $n$ and $x=T y=s y$.

So, we define a semi compatible pair of self-maps in fuzzy metric space by condition (ii) only. and [3] proved some interesting fixed point results using implicit real functions and semi compatibility in $d$-complete topological spaces. Recently, Popa in used the family $F 4$ of implicit real functions to find the fixed points of two pairs of semi-compatible maps in a $d$-complete topological space. Here, $F 4$ denotes the family of all real continuous functions Popa introduced the idea of implicit function to prove a common fixed point theorem in metric spaces . Recently in 2009, using the concept of subcompatible maps. [10] proved common fixed point theorems. In 2010 and 2011, [1], [4], [8],

Manuscript received January 8, 2013; revised March 25, 2013.

Sonal Dani is with the Department of Applied Mathematics Chhatrapati Shivaji Institute of Technology, Durg(C.G) India (e-mail: sonaldani18@gmail.com).

Sanjay Sharma is with the Department of Applied Mathematics Bhilai Institute of Technology, $\operatorname{Durg}(\mathrm{C} . \mathrm{G})$ India (e-mail: ssharma_bit@yahoo.co.in). proved fixed point theorems in Fuzzy metric space.

\section{PRELIMINARIES}

Definition 2.1. A Fixed point of a function is a point that is mapped to itself by the function .

A set of fixed point is some time called fixed set, that is to say , $c$ is fixed point of function $f(x)$ if and only if $f(c)=c$. If $f(x)$ is defined by real number by $f(x)=$ $x^{2}-3 x+4$ then 2 is fixed point of $\mathrm{f}$ because $f(2)=2$

Let $X$ be a set and let $T: X \rightarrow X$ be a function that maps $X$ into itself. A fixed point $T$ is an element $x \in X$ for which $T(x)=x$

Example Let $X$ be the two elements set $\{\mathrm{a}, \mathrm{b}\}$.The function $F: X \rightarrow X$ defined by

$F(a)=b$ and $F(b)=a$ has no fixed point, but the other three function that maps $X$ into itself each have one or two fixed point.

Definition 2.2. Let $X$ be any non empty set. A fuzzy set $M$ in $X$ is a function with domain $X$ and values in $[0,1]$.

Definition 2.3. A binary operation $*$ : $[0,1]$ $\times[0,1] \rightarrow[0,1]$ is a continuous t-norm if it satisfy the following condition:

1) * is associative and commutative

2) * is continous function.

3) $a * 1=a$ for all $\quad a \in[0,1]$

4) $a * b \leq c * d$ whenever $a \leq c$ and $b \leq d$ and $a, b, c, d \in[0,1]$

Examples of t-norms are $a * b=a b$ and $a * b=\min \{a, b\}$

Definition 2.4 . The 3-tuple $(X, M, *)$ is called a fuzzy metric space if $X$ is an arbitrary set, $*$ is a continuous $t$-norm, and $M$ is a fuzzy set in $X^{2} \times[0, \infty[$ satisfying the following conditions for all $x, y, z \in X$ and $s, t>0$

1) $M(x, y, 0)=0$

2) $\quad M(x, y, t)=1$, for all $t>0$ if and only if $x=y$;

3) $M(x, y, t)=M(y, x, t)$

4) $\quad M(x, y, t) * M(y, z, s) \geq M(x, z, t+s)$ 
5) $\quad M(x, y, \cdot):[0, \infty[\rightarrow[0,1]$ is left continuous.

Definition 2.5. A sequence $\left\{x_{n}\right\}$ in a Fuzzy metric space $(X, M, *)$ is said to be a Cauchy sequence if and only if for each $\in>0, t>0$, there exists $\mathrm{n}_{0} \in \mathrm{N}$ such that $\left.M\left(x_{n}, x_{m}, t\right)\right\rangle 1-\varepsilon$ for all $n, m \geq n_{0}$.

The sequence is said to converge to a point $x$ in $X$ if and only if for each $\varepsilon>0, t>o$, there exists $n_{0} \in N$ such that $\left.M\left(x_{n}, x_{m}, t\right)\right\rangle 1-\varepsilon$ for all $n \geq n_{0}$.

A Fuzzy metric space $(X, M, *)$ is said to be complete if every Cauchy sequence in it converges to a point in it.

Definition 2.6. Two maps $A$ and $S$ from a fuzzy metric space $(X, M, *)$ into itself are said to be $\mathrm{R}$-weakly commuting if there exists a positive real number $\mathrm{R}$ such that for each $x \in X$

$$
\lim _{n \rightarrow \infty} M(A S x, S A x, R t) \geq M(A x, S x, t)
$$

for all $t>0$.

Definition 2.7. Two maps $A$ and $B$ from a fuzzy metric space $(X, M, *)$ into itself are said to be compatible if $\lim _{n \rightarrow \infty} M\left(A B x_{n}, B A x_{n}, t\right)=1$ for all $t>0$, when ever $\left\{x_{n}\right\}$ is a sequence such that $\lim _{n \rightarrow \infty} A x_{n}=\lim _{n \rightarrow \infty} B x_{n}$ for some $x \in X$.

Definition 2.8. Let $A$ and $S$ be mappings from a fuzzy metric space $(X, M, *)$ into itself.

Then the mappings are said to be semi compatible if $\lim _{n \rightarrow \infty} M\left(A S x_{n}, S x, t\right)=1$, for all $t>0$ whenever $\left\{x_{n}\right\}$ is $A(x)$ a sequence in $X$ such that $\lim _{n \rightarrow \infty} A x_{n}=\lim _{n \rightarrow \infty} S x_{n}=x$ It follows thatif $(A, S)$ is semicompatible and $A y=S y$, then $A S y=A S y$. Thus if the pair $(A, S)$ is semicompatible.

Definition 2.9: Let be a fuzzy metric $\operatorname{space}(X, M, *)$ and $f: X \rightarrow X$ and $F: X \rightarrow B(X)$. . Then $(f, F)$ is said to be a pair of M-maps with respect $f$ to if there exists a sequence $\left\{x_{n}\right\}$ in $X$ such that for every $t>0$,

$$
M\left(f x_{n}, z, t\right) \rightarrow 1 \text { and } \partial_{m}(F x,\{z\}, t) \rightarrow 1
$$

as $n \rightarrow \infty$ for some $z \in f(x)$

Definition 2.10: Let be a fuzzy metric $\operatorname{space}(X, M, *)$ and $f, g: X \rightarrow X$ and $F: X \rightarrow B(X)$. . Then $(f, F)$ is said to be a pair of M-maps with respect to $(f, F)$ if there exists a sequence $\left\{x_{n}\right\}$ in $\mathrm{X}$ such that for every $t>0$,

$$
M\left(f x_{n}, z, t\right) \rightarrow 1 \text { and } \partial_{m}(F x,\{z\}, t) \rightarrow 1
$$

as $n \rightarrow \infty$ for some $z \in f(x) \cap g(x)$.

Definition 2.11. Self mappings $A$ and $S$ of a Fuzzy metric space $(X, M, *)$ said to be sub compatible if there exists a sequence $\left\{x_{n}\right\}$ in $X$ such that that $\lim _{n \rightarrow \infty} A x_{n}=\lim _{n \rightarrow \infty} S x_{n} \quad, x \in X \quad$ and $\quad$ satisfy $\lim _{n \rightarrow \infty} M\left(A S x_{n}, S A x_{n}, t\right)=1$.

\section{MAIN RESUlT}

Theorem : Let $A, B, S, T$ be self maps on complete fuzzy metric space $(X, M, *)$ satisfying

$$
\phi\left\{\begin{array}{l}
\partial_{m}(S x, T y, K t), M(A x, B y, t), \partial_{m}(A x, S x, t) \\
\partial_{m}(B y, T y, t), \partial_{m}(A x, T y, t), \partial_{m}(B y, S x, t)
\end{array}\right\} \geq 0
$$

for all $x, y \in X, t>0$ and $k \in(0,1)$ where $\phi \in \Phi$

1) $(A, S)$ is a pair of M-maps with respect to $A$ and $S x \subseteq B(x)$ for all $x \in X$

2) $(B, T)$ is a pair of M-maps with respect to $B$ and $B x \subseteq A(x)$ for all $x \in X$

3) $(A, S)$ is semi compatible and $(B, T)$ is weak compatible.

4) $(A, S)$ and $(B, T)$ is sub compatible.

Then $A, B, S$, and $T$ have a unique common fixed point $z \in X$ such that

$$
S z=T z=\{z\}=\{A z\}=\{B z\} .
$$

Proof: Suppose (1) is holds.

Since $(A, S)$ is a pair of M-maps with respect to $A$ and $(A, S)$ is semi compatible, there exists a sequence $\left\{x_{n}\right\}$ in $X$ such that for every $t>0, \lim _{n \rightarrow \infty} M\left(A x_{n}, z, t\right)=1$ and $\lim _{n \rightarrow \infty} \partial_{m}\left(A x_{n},\{z\}, t\right)=1$ for some $z \in A(X)$

Hence there exists $w \in X$ such that $z=A w$.

Since $S x \subseteq B(x)$ for all $x \in X$ there exist $\mu_{n} \in S x_{n}$ and $y_{n} \in X$ such that $\mu_{n}=B y_{n}$ for all $n$.

Also $M\left(B y_{n}, z, t\right)=M\left(\mu_{n}, z, t\right) \geq \partial_{m}\left(S x_{n},\{z\}, t\right)$ $\rightarrow 1 n \rightarrow \infty$

Hence $\lim _{n \rightarrow \infty} M\left(B y_{n}, z, t\right)=1$

$$
\phi\left\{\begin{array}{l}
\partial_{m}\left(S x_{n}, T y_{n}, K t\right), M\left(A x_{n}, B y_{n}, t\right), \partial_{m}\left(A x_{n}, S x_{n}, t\right) \\
\partial_{m}\left(B y_{n}, T y_{n}, t\right), \partial_{m}\left(A x_{n}, T y_{n}, t\right), \partial_{m}\left(B y_{n}, S x_{n}, t\right)
\end{array}\right\} \geq 0
$$


Letting $n \rightarrow \infty$ we have,

$\phi\left(\partial_{m}\left(\{z\}, \lim _{n \rightarrow \infty} T y_{n}, K t\right), 1,1, \partial_{m}\left(\{z\}, \lim _{n \rightarrow \infty} T y_{n}, t\right), \partial_{m}\left(\{z\}, \lim _{n \rightarrow \infty} T y_{n}, t\right), 1\right) \geq 0$

Hence

$$
\partial_{m}\left(\{z\}, \lim _{n \rightarrow \infty} T y_{n}, K t\right) \quad \geq \partial_{m}\left(\{z\}, \lim _{n \rightarrow \infty} T y_{n}, t\right) \text { from }
$$

property of $\phi \in \Phi$

We have if $\left\{G_{n}\right\}$ and $\left\{F_{n}\right\}$ are sequences in $H(x)$ which is non empty bounded subset of fuzzy metric space $(X, M, *)$ converging to $G$ and $F$ in $H(x)$, respectively. Then $\partial_{m}\left(G_{n}, F_{n}, t\right) \rightarrow \partial_{m}(G, F, t)$ as $n \rightarrow \infty$ for all $t>0$. And if $\partial_{m}\left(G_{n}, F_{n}, t\right) \rightarrow \partial_{m}(G, F, t)$. For all $G, F \in H(X)$ and for all $t>0,0<k<1$ then $G=F=\{$ singleton $\}$ provided $M(x, y, t) \rightarrow 1$ as $t \rightarrow \infty$ for all $x, y \in X$

From above condition we have $\lim _{n \rightarrow \infty} T y_{n}=\{z\}$.

Thus $\lim _{n \rightarrow \infty} S x_{n}=\lim _{n \rightarrow \infty} T y_{n}=\lim _{n \rightarrow \infty} A x_{n}=\lim _{n \rightarrow \infty} B y_{n}=$ $\{z\}(A, S)$ is semi compatible)

Now,

$\phi\left\{\begin{array}{l}\partial_{m}\left(S w, T y_{n}, K t\right), M\left(A w, B y_{n}, t\right), \partial_{m}(A w, S w, t) \\ \partial_{m}\left(B y_{n}, T y_{n}, t\right), \partial_{m}\left(A w, T y_{n}, t\right), \partial_{m}\left(B y_{n}, S w, t\right)\end{array}\right\} \geq 0$

Letting $n \rightarrow \infty$ we have,

$\phi\left(\partial_{m}(S w,\{z\}, K t), 1, \partial_{m}(\{z\}, S w, t), 1,1, \partial_{m}(\{z\}, S w, t)\right) \geq 0$

$\partial_{m}(S w,\{z\}, K t) \geq \partial_{m}(S w,\{z\}, K t)$

Hence $S w=\{z\}$ Thus $S w=\{z\}=\{A w\}$

Since $\{z\}=S w \subseteq B(X)$, there exist $\gamma \in X$ such that $z=B \gamma$

Now,

$\phi\left\{\begin{array}{l}\partial_{m}\left(S x_{n}, T \gamma, K t\right), M\left(A x_{n}, B \gamma, t\right), \partial_{m}\left(A x_{n}, S x_{n}, t\right) \\ \partial_{m}(B \gamma, T \gamma, t), \partial_{m}\left(A x_{n}, T \gamma, t\right), \partial_{m}\left(B \gamma, S x_{n}, t\right)\end{array}\right\} \geq 0$

Letting $n \rightarrow \infty$ we have,

$\phi\left(\partial_{m}(\{z\}, T \gamma, K t), 1,1, \partial_{m}(\{z\}, T \gamma, t), \partial_{m}(\{z\}, T \gamma, t), 1\right) \geq 0$

$$
\partial_{m}(\{z\}, T \gamma, K t) \geq \partial_{m}(\{z\}, T \gamma, t)
$$

Hence $T \gamma=\{z\}$. Thus $T \gamma=\{z\}=\{B \gamma\} .(B, T)$ is weak compatible.

Since the pair $(A, S)$ and $(B, T)$ are sub compatible, we have

$$
S z=\{A z\} . \text { and } T z=\{B z\}
$$

$\phi\left\{\begin{array}{l}\partial_{m}(S z, T \gamma, K t), M(A z, B \gamma, t), \partial_{m}(A z, S z, t) \\ \partial_{m}(B \gamma, T \gamma, t), \partial_{m}(A z, T \gamma, t), \partial_{m}(B \gamma, S z, t)\end{array}\right\} \geq 0$

$\phi(M(A z, z, K t), M(A z, z, t), 1,1 M(A z, z, t) M(z, A z, t)) \geq 0$

$$
M(A z, z, k t) \geq M(A z, z, t) .
$$

Hence $A z=z$ Thus $S z=\{A z\}=\{z\}$

Also,

$\phi\left\{\begin{array}{l}\partial_{m}(S w, T z, K t), M(A w, B z, t), \partial_{m}(A w, S w, t) \\ \partial_{m}(B z, T z, t), \partial_{m}(A w, T z, t), \partial_{m}(B z, S w, t)\end{array}\right\} \geq 0$

$\phi(M(z, B z, K t), M(z, B z, t), 1,1 M(z, B z, t) M(B z, z, t)) \geq 0$

$$
M(z, B z, k t) \geq M(z, B z, t) .
$$

Hence $B z=z$ Thus $T z=\{B z\}=\{z\}$.

Thus $z$ is a common fixed point of $A, B, S, T$ such that $S z=T z=\{z\}=\{A z\}=\{B z\}$. Uniqueness of common fixed point follows from.

\section{REFERENCES}

[1] I. Kramosil and J. Michalek, "Fuzzy metric and statistical metric spaces," Kybernetika, vol. 11, pp. 326-334, 1975.

[2] A. George and P. Veeramani, "On some results in fuzzy metric spaces," Fuzzy sets and systems, vol. 64, pp. 395-399, 1994.

[3] M. Grabiec, "Fixed points in fuzzy metric space," Fuzzy sets and systems, vol. 27, pp. 385-389, 1988.

[4] S. N. Mishara, N. Shrma, and S. L. Singh, "Common fixed points of maps in fuzzy metric spaces, Internat," J. Math. Sci., vol. 29, no. 3, pp. 227-238, 1998.

[5] I. Altun and D. Turkoglu, "Some fixed point theorems on fuzzy metric spaces with implicit relations," Commun. Korean. Math. Soc., vol. 23, no. 1, pp. 111-124, 2008

[6] Y. J. Cho, S. Sedghi, and N. Shobe, "Generalized fixed point theorems for compatible mappings with types in fuzzy metric spaces," Chaos, Solitons and fractals, vol. 39, pp. 2233-2244, 2009.

[7] A. Mujahid, A. Ishak, and G. Dhananjay, "Common fixed point theorems for non compatible mappings in fuzzy metric spaces," Bulletin of Mathematical Analysis and Applications, vol. 1, issue 2, pp. 47-56, 2009.

[8] S. Kumar and B. Fisher, "A common fixed point theorem in fuzzy metric space using property(E.A) and implicitrelation," Thai Journal of Mathematics, vol. 8, no. 3, pp. 439-446, 2010.

[9] Y. J. Cho, B. K. Sharma, and D. R. Sahu, "Semi-compatibility and fixed points," Math. Japon., vol. 42, no. 1, pp. 91-98, 1995.

[10] H. Bouhadjera and C. Godet-Thobie, "Common fixed point theorems for pairs of subcompatible maps," arXiv:0906.3159v1 [math.FA], vol. 17, June 2009.

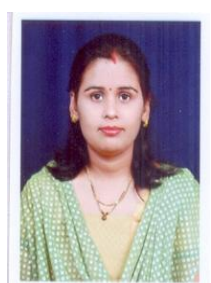

Sonal Dani was born in India on Oct. 18, 1979. Sonal Dani passed her M.Sc (mathematics) from Chhattisgarh college Raipur,India. And M. Phil degree fromVenaykamission, selam, Tamilnadu, India in year 2009. Presently she is working as Research scholar under Dr. Sanjay sharma..

She is working as Assistant professor in Chhattrapati Shivaji Institute of Technology, Durg India..she published articles: 1) Sonal D and S. Sharma, Weak Compatibility in fuzzy metric space IJAMA: 4(2), December 2012, pp. 115-120. 2) Sonal D and S. Sharma,Fuzzy metric space for non compatible maps International Journal of Pure and Applied Mathematics vol. 83 no. 2 2013, 295-301. 3) Sonal D and S. Sharma Common Fixed Point Theorem for Non Compatible Maps International Journal of Mathematics Research.Volume 4, Number 6 (2012), pp. 761-766. 